\title{
An Improvement Proposal to the Static Friction Model
}

\author{
Sergio Sánchez-Mazuca and Ricardo Campa \\ Division of Graduate Studies and Research, Technological Institute of La Laguna, 27000 Torreón, COAH, Mexico \\ Correspondence should be addressed to Ricardo Campa; recampa@itlalaguna.edu.mx
}

Received 17 March 2013; Accepted 20 May 2013

Academic Editor: Qingsong Xu

Copyright (c) 2013 S. Sánchez-Mazuca and R. Campa. This is an open access article distributed under the Creative Commons Attribution License, which permits unrestricted use, distribution, and reproduction in any medium, provided the original work is properly cited.

\begin{abstract}
Friction is a force acting against the relative motion between two surfaces in contact. This phenomenon is present in all mechanical systems and has a great impact on the control area. The design of mechatronic systems and the compensation techniques require a broad knowledge of the effects that friction produces. The phenomenon has two well-defined phases: static friction presents before the motion between the surfaces in contact is clearly visible, while kinetic friction appears when that motion at large scale has already started. There are different friction models for each of those phases. In this work we propose an improvement to the static friction models, which consist in assuming that the maximum static friction coefficient is no more a constant but a function of the rate of change of the external force that produces the motion. After explaining and justifying the proposal, the procedure for obtaining the parameters of the new model is mentioned. At the end, an experimental study on a direct-drive motor allows us to validate the proposed model.
\end{abstract}

\section{Introduction}

The growing interest of the robotics community in friction modelling and compensation is due to the requirement of more precision in robot positioning.

Friction affects the performance of the systems that control a mechanism, producing positioning errors during the execution of a given task. By better understanding the friction phenomenon it becomes easier to design control laws for reducing its undesired effects.

Throughout the time, several models have been proposed, each trying to capture the essence of the complicated phenomenon of friction; however, finding a model to describe all its effects is difficult [1]. Friction models can be either static (without memory) or dynamic (with memory), and they can be described by algebraic and/or differential equations or be based on events.

In general terms, the static friction (also known by the portmanteau stiction) is the force that opposes to the start of a visible motion between two surfaces in contact. In the past it was thought that, during the static friction phase (i.e., before reaching the so-called breakaway point), there was no motion at all between the surfaces in contact; now, after the work of [2], it is known that, before the breakaway point, there can be very small displacements (or microdisplacements) and other friction effects such as that known as "stick-slip" [3], which make the static friction phase difficult to model. On the other hand, the kinetic friction phase starts once the breakaway point is reached, and a visible motion is present.

The so-called maximum static friction coefficient indicates the maximum value of the friction force at which the breakaway point occurs; this coefficient is considered a constant in practically all the friction models.

The main contribution of this work is precisely to propose a modification to the models including the maximum static friction coefficient, by no more considering it as a constant but a function of the rate of change of the external force applied between the surfaces in contact. This proposal is based on observations made during a series of experiments carried out on a direct-drive brushless DC (BLDC) rotary motor. Similar observations were reported by Johannes et al. [4] in different systems with friction, but in that work no explicit expression for such function was proposed.

It is worth mentioning here that, in the case of a rotary motion, friction is no longer a force but a torque. Nevertheless, for all purposes, the behavior of friction is identical in linear, and rotary motion. 
One important characteristic of BLDC motors is that they are controlled using a drive which can be configured so that its input signal is proportional to either the torque or the velocity of the motor (thus defining, resp., the so-called "torque mode" and "velocity mode" for the operation of the drive). That is why this kind of motors is commonly used as actuators in robots and machine tools. More information on modelling and parameter identification of BLDC motors can be found in [5].

The remainder of this paper is organized as follows. The most common models including the static friction effect are described in Section 2. The proposed modification to the static friction model is presented in Section 3. The procedure to estimate the parameters of the proposed model is given in Section 4, while Section 5 describes both the application of such procedure to obtain the parameters and the experimental validation of the proposed modification in the studied motor. Finally, in Section 6, some concluding remarks are given.

\section{Friction Models}

As mentioned before, friction is generally divided into static friction and kinetic friction; the former corresponds to all the effects which are present without a visible motion, while the latter refers to everything which happens once that motion exists. Nevertheless, after the results on microdisplacements presented in [2], now it is common to talk about the presliding phase (where effects such as maximum static friction and microdisplacements occur) and the sliding phase (where Coulomb friction, viscous friction, and the Stribeck effect are present).

Consider a motor with friction torque $f$; let $q, \dot{q}$, and $\ddot{q}$ be, respectively, the angular displacement, velocity, and acceleration of the motor shaft; also, let $\tau$ be the external torque applied to the motor to produce the motion. The relation among these quantities is given by the motor dynamics

$$
J \ddot{q}+f=\tau,
$$

where $J$ stands for the moment of inertia of the motor.

It is common to assume that the friction torque of a motor depends only on the velocity, that is, $f(\dot{q})$. However, in 1833, Morin [6] established the first model for static friction, stating that such friction can be described satisfactorily not only as a function of velocity but also of the applied torque. Thus, for a motor, we can write, in general, $f(\dot{q}, \tau)$.

2.1. Static (Memory-Less) Models. Morin's static friction model [6] is defined for $\dot{q}=0$ and is given by

$$
f(0, \tau)= \begin{cases}\tau, & \text { if }|\tau|<f_{s}, \\ f_{s} \operatorname{sgn}(\tau), & \text { if }|\tau| \geq f_{s},\end{cases}
$$

where the sign function is defined as

$$
\operatorname{sgn}(x)= \begin{cases}1, & \text { if } x>0 \\ 0, & \text { if } x=0 \\ -1, & \text { if } x<0\end{cases}
$$

and $f_{s}$ is the maximum static friction coefficient, which is assumed to be constant.

With respect to kinetic friction, it is present when $\dot{q} \neq 0$ and does not depend on $\tau$, so it is denoted as $f_{k}(\dot{q})$. One of the most common models for describing kinetic friction is the one combining the Coulomb friction and the viscous friction effects; this model can be written as

$$
f_{k}(\dot{q})=f_{c} \operatorname{sgn}(\dot{q})+f_{v} \dot{q}
$$

where $f_{c}$ and $f_{v}$ are the Coulomb friction and viscous friction coefficients, respectively. Note in (4) that, while viscous friction (second term in the right-hand side) is proportional to $\dot{q}$, Coulomb friction (first term) has the same sign as $\dot{q}$ but a constant magnitude (equal to $f_{c}$ ).

In 1902 Stribeck carried out some experiments at a constant velocity of low amplitude [7], showing the existence of the phenomenon now known as Stribeck effect, that describes the transition between the maximum static friction and the Coulomb friction. One of the expressions more used to model this phenomenon is the following exponential function known as the Armstrong model [8]:

$$
f_{k}(\dot{q})=\operatorname{sgn}(\dot{q})\left[f_{c}+\left(f_{s}-f_{c}\right) e^{-\left|\dot{q} / v_{s}\right|^{\delta_{v}}}\right]+f_{v} \dot{q}
$$

which incorporates the static friction, Coulomb friction, and viscous friction coefficients and includes two more parameters: the Stribeck velocity coefficient $v_{s}$ and a fitting parameter $\delta_{v}$.

Figure 1 shows the graphs of friction against velocity of the kinetic friction given by (4) and (5). The static friction model (2) can be combined with kinetic friction in the following way:

$$
f(\dot{q}, \tau)= \begin{cases}\tau, & \text { if } \dot{q}=0,|\tau|<f_{s}, \\ f_{s} \operatorname{sgn}(\tau), & \text { if } \dot{q}=0,|\tau| \geq f_{s}, \\ f_{k}(\dot{q}), & \text { if } \dot{q} \neq 0,\end{cases}
$$

where $f_{k}(\dot{q})$ can be $(4),(5)$, or any other kinetic friction model (with $\dot{q} \neq 0$ ). Note that (6) can also be written as

$$
f(\dot{q}, \tau)= \begin{cases}\operatorname{sat}\left(\tau, f_{s}\right), & \text { if } \dot{q}=0, \\ f_{k}(\dot{q}), & \text { if } \dot{q} \neq 0,\end{cases}
$$

where the saturation function $\operatorname{sat}(x, a)$ is defined as

$$
\operatorname{sat}(x, a)= \begin{cases}x, & \text { if }|x|<a, \\ a \operatorname{sgn}(x), & \text { if }|x| \geq a .\end{cases}
$$

2.2. Dynamic Models. Friction is a complex phenomenon that cannot be completely described with the static models mentioned above. Experimental evidence has shown that friction has its own dynamics, with memory, mainly at the low velocity region; for that reason, it is necessary to use differential equations to model its behavior. Two of the more important dynamic friction models found in literature are recalled below. 


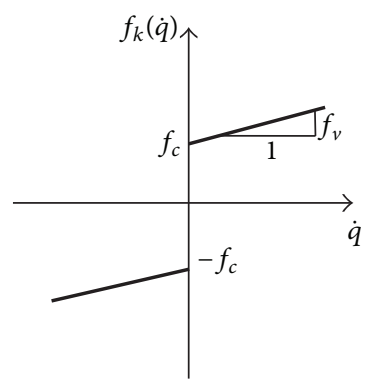

(a)

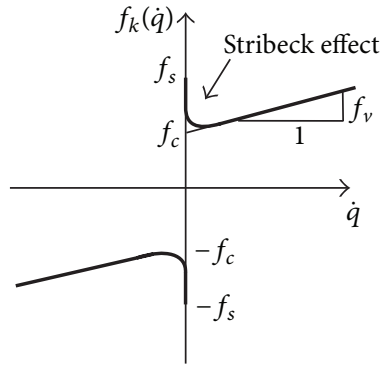

(b)

FIGURE 1: Friction torque versus velocity for static models: (a) Coulomb plus viscous friction model (4); (b) Armstrong model (5).

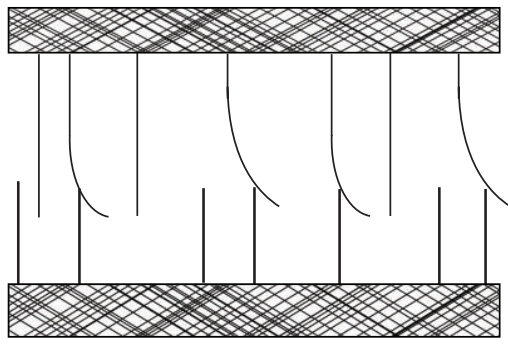

(a)

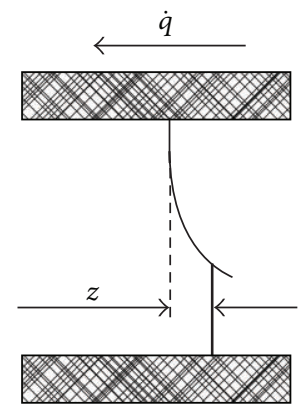

(b)

Figure 2: Dahl model: (a) as two brushes, facing each other; (b) equivalent average deflection of the brushes.

2.2.1. Dahl Model. In 1968 Dahl proposed a dynamic friction model based on the behavior of the roughness between two surfaces in contact [9]. Dahl considers that this roughness can be modeled as the bristles of two brushes (see Figure 2); thus, if $z$ represents the average deflection of the bristles, then $z$ times a stiffness coefficient $\sigma_{0}$ gives the static friction torque. The two key features of the Dahl model are the following.

(i) The $z$ variable has its own dynamics, which depends on the actual velocity of the motor.

(ii) Static friction (with $\dot{q}=0$ ) is bounded and belongs to the interval $\left[-f_{c}, f_{c}\right]$; in other words, $\left|\sigma_{0} z\right| \leq f_{c}$ or, equivalently, $|z| \leq f_{c} / \sigma_{0}$.

If viscous friction effect is included, then the Dahl model is given by

$$
\begin{gathered}
f(\dot{q}, z)=\sigma_{0} z+f_{v} \dot{q}, \\
\dot{z}=-\frac{\sigma_{0}|\dot{q}|}{f_{c}} z+\dot{q},
\end{gathered}
$$

where (10) gives the dynamics of the variable $z$.

Now let us consider the case where the applied torque $\tau=$ $\tau_{0}$ is a constant. It could be noticed from (9) that

(i) if $\dot{q}=0$ and $\left|\tau_{0}\right| \leq f_{c}$, then $\dot{z}=0$, and $z$ stays at a constant value, not necessarily zero; from (1) and (9), that constant value must be $z=f / \sigma_{0}=\tau_{0} / \sigma_{0}$, and such condition corresponds to the equilibrium state of the system defined by (1), (9), and (10); (ii) if $\dot{q} \neq 0$ and $\left|\tau_{0}\right| \leq f_{c}$, then $z$ (and $f$ ) changes its value opposing to the motion and reducing $|\dot{q}|$ to zero, that is, reaching the equilibrium state;

(iii) if $\left|\tau_{0}\right|>f_{c}$, then it is not possible to reach the equilibrium state; both $|\dot{q}|$ and $|z|$ increase up to their corresponding steady-state values, $\left|\dot{q}_{s s}\right|$ and $\left|z_{s s}\right|$, respectively, where $\ddot{q}=0$ and $\dot{z}=0$; moreover, it is easy to show that $\left|z_{s s}\right|=f_{c} / \sigma_{0}$ and $\left|\dot{q}_{s s}\right|=\left(\tau_{0}-f_{c}\right) / f_{v}$.

In summary, the two phases of friction are clearly defined for Dahl model; if $|\tau|<f_{c}$, then it is always possible to reach a state for which $\dot{q}=0, z=\tau / \sigma_{0}$, and $f(0, z)=\tau$; on the other hand, if $|\tau|>f_{c}$, then $\dot{q}=\dot{q}_{s s} \neq 0, z=z_{s s}=\left(f_{c} / \sigma_{0}\right) \operatorname{sgn}(\dot{q})$, and $f\left(\dot{q}_{s s}, z_{s s}\right)=f_{c} \operatorname{sgn}\left(\dot{q}_{s s}\right)+f_{v} \dot{q}_{s s}$. Thus the Dahl model (9) and (10) gives a smooth approximation of model (4), which is discontinuous.

2.2.2. LuGre Model. In 1995 researchers of the Lund Institute of Technology, in Sweden, and the Institut National Polytechnique of Grenoble, in France, developed the so-called LuGre model (from Lund and Grenoble), which is one of the most complete friction models nowadays [10]. The LuGre model is a generalization of the Dahl model, which considers not only the presliding phenomenon but also the Stribeck effect. As the Dahl model, the LuGre model can be expressed in terms of the velocity $\dot{q}$ and the average bristle deflection $z$; but the latter also includes a term proportional to $\dot{z}$, that is

$$
f(\dot{q}, z)=\sigma_{0} z+\sigma_{1} \dot{z}+f_{v} \dot{q}
$$


where $\sigma_{1}$ is the damping coefficient of the bristles and the dynamics of $z$ is now

$$
\dot{z}=-\frac{\sigma_{0}|\dot{q}|}{g(\dot{q})} z+\dot{q},
$$

with

$$
g(\dot{q})=f_{c}+\left(f_{s}-f_{c}\right) e^{-\mid \dot{q} / v_{s} \delta_{s}^{\delta_{s}}} ;
$$

the function $g(\dot{q})$ allows to extend Dahl model to describe Stribeck effect, employing the same exponential function as in (5). Notice that in this model, as $f_{c} \leq g(\dot{q}) \leq f_{s}$, then $|z| \leq f_{s} / \sigma_{0}$.

An analysis similar to that in Section 2.2.1 can be done with the LuGre model. Considering again a constant input torque $\tau=\tau_{0}$ we have the three following cases.

(i) If $\left|\tau_{0}\right| \leq f_{c}$, then it is always possible to reach an equilibrium where $\dot{q}=0$ and $z=\tau_{0} / \sigma_{0}$.

(ii) If $\left|\tau_{0}\right|>f_{s}$ then the equilibrium cannot be reached, but the states $\dot{q}$ and $z$ tend to their steady-state values $\dot{q}_{s s}$ and $z_{s s}$, respectively, where, for a given $\dot{q}_{s s}$, we have that $\left|z_{s s}\right|=g\left(\dot{q}_{s s}\right) / \sigma_{0}$.

(iii) If $f_{c}<\left|\tau_{0}\right| \leq f_{s}$, then the states $\dot{q}$ and $z$ can reach either the equilibrium or the steady-state $\dot{q}_{s s}$ and $z_{s s}$, depending on the initial conditions.

It is easy to show that the friction torque in the steady state is given by $f\left(\dot{q}_{s s}\right)=g\left(\dot{q}_{s s}\right) \operatorname{sgn}\left(\dot{q}_{s s}\right)+f_{v} \dot{q}_{s s}$, so the LuGre model is related to Armstrong model (5).

Figure 3 shows the graphs of friction versus velocity for the Dahl model (9) and (10) and the LuGre model (11)-(13). Notice the existence of microdisplacements.

\section{Improvement Proposal}

The maximum static friction coefficient $f_{s}$ indicates the maximum breakaway torque, that is, the maximum torque that can be applied to a motor at rest, before it starts a visible motion. Most of friction models assume that this coefficient is constant. For its estimation, it is suggested to apply a ramp of input torque with very small slope $m$; that is, $\tau=m t$ [11].

Nevertheless, it is a known fact that the breakaway torque depends on the value of $m$; the smaller the value of $m$, the higher the breakaway torque. This dependence was first observed in [4], where a graph as the one shown in Figure 4 is presented. That graph indicates how the breakaway force, $\phi_{s}$, varies as a function of the rate of change of the applied torque $\dot{\tau}$ (notice that, if $\tau=m t$, then $\dot{\tau}=m$ ). However, no attempt is made in [4] to characterize such a function.

One of the main contributions of our work is precisely to introduce an explicit expression for the variation of the breakaway torque as a function of the rate of change of the applied torque.

After several experiments on a BLDC servomotor, we concluded that for torque inputs with a small rate of change (i.e., $|\dot{\boldsymbol{\tau}}| \rightarrow 0$ ) the breakaway force approximates the value of $f_{s}$. On the other hand, as $\dot{\tau}$ increases $(|\dot{\tau}| \rightarrow \infty)$, the value of the breakaway force is near $f_{c}$. We then propose the following expression:

$$
\phi_{s}(\dot{\tau})=f_{c}+\left(f_{s}-f_{c}\right) e^{-\mid \dot{\tau} / t_{s} \delta^{\delta_{t}}},
$$

where $f_{s}$ and $f_{c}$ are already defined, $t_{s}$ is called here the torque rate coefficient, and $\delta_{t}$ is a curve fitting parameter. Note the similarity between expressions (14), describing the variation of the breakaway torque with $\dot{\tau}$, and the exponential function in (5), indicating the kinetic friction at low velocities.

Analysing (14) it is observed that if the applied torque is constant (i.e., if $\dot{\tau}=0$ ), then the breakaway torque is

$$
\phi_{s}(0)=f_{s} .
$$

On the other hand, if $\tau$ changes abruptly (i.e., if $\dot{\tau} \rightarrow \infty$ ), as when a torque step is applied to the motor, then,

$$
\lim _{\dot{\tau} \rightarrow \infty}\left|\phi_{s}(\dot{\tau})\right|=f_{c} .
$$

And for all $\dot{\tau}$ we have

$$
f_{c} \leq \phi_{s}(\dot{\tau}) \leq f_{s} .
$$

Most of the friction models do not consider such a variation of the breakaway torque; they only take into account the maximum breakaway torque, given by $f_{s}$. Our proposal is to replace the maximum static friction coefficient $f_{s}$ by $\phi_{s}(\dot{\tau})$ in those friction models including such parameter. As a result we get new models for which friction depends not only on $\dot{q}$ and $\tau$ but also on $\dot{\tau}$.

As an example, let us consider the Armstrong model (5) and replace $f_{s}$ by $\phi_{s}(\dot{\tau})$ to get

$$
f_{k}(\dot{q}, \dot{\tau})=\operatorname{sgn}(\dot{q})\left[f_{c}+\left(\phi_{s}(\dot{\tau})-f_{c}\right) e^{-\mid \dot{q} / v_{s} \delta_{v}^{\delta_{v}}}\right]+f_{v} \dot{q} ;
$$

then, substituting (14) and simplifying

$$
f_{k}(\dot{q}, \dot{\tau})=\operatorname{sgn}(\dot{q})\left[f_{c}+\left(f_{s}-f_{c}\right) e^{-\left(\left|\dot{q} / v_{s} \delta^{\delta_{v}}+\right| \dot{\tau} /\left.t_{s}\right|^{\delta_{t}}\right)}\right]+f_{v} \dot{q} .
$$

Figure 5 shows a 3D graph of function (19). Notice that the curve in Figure 1(b) for the Armstrong model corresponds to the cross-section of Figure 5, where $\dot{\tau}=0$.

Moreover, the kinetic friction model (19) can also be combined with the static friction model (2) as in (7) but replacing $f_{s}$ by $\phi_{s}(\dot{\tau})$; that is,

$$
f(\dot{q}, \tau, \dot{\tau})= \begin{cases}\operatorname{sat}\left(\tau, \phi_{s}(\dot{\tau})\right), & \text { if } \dot{q}=0, \\ f_{k}(\dot{q}, \dot{\tau}), & \text { if } \dot{q} \neq 0 .\end{cases}
$$

Now, let us consider the LuGre model (11)-(13) with $\phi_{s}(\dot{\tau})$ in (14) instead of $f_{s}$, we get

$$
\begin{gathered}
f(\dot{q}, z, \dot{\tau})=\sigma_{0} z+\sigma_{1} \dot{z}+f_{v} \dot{q}, \\
\dot{z}=-\frac{\sigma_{0}|\dot{q}|}{g(\dot{q}, \dot{\tau})} z+\dot{q}, \\
g(\dot{q}, \dot{\tau})=f_{c}+\left(f_{s}-f_{c}\right) e^{-\left(\left|\dot{\tau} / t_{s}\right|^{\delta_{t}}+\left|\dot{q} / v_{s}\right|^{\delta_{v}}\right.} .
\end{gathered}
$$




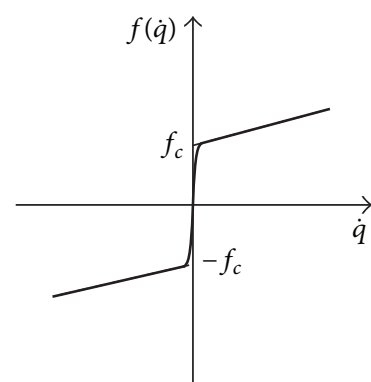

(a)

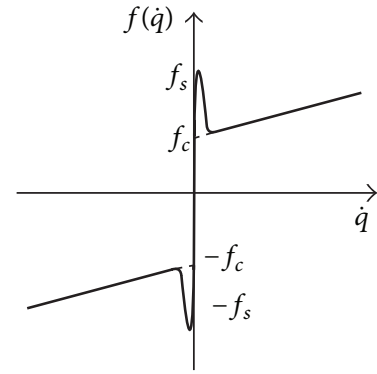

(b)

FIGURE 3: Friction torque versus velocity for dynamic models: (a) Dahl model; (b) LuGre model.

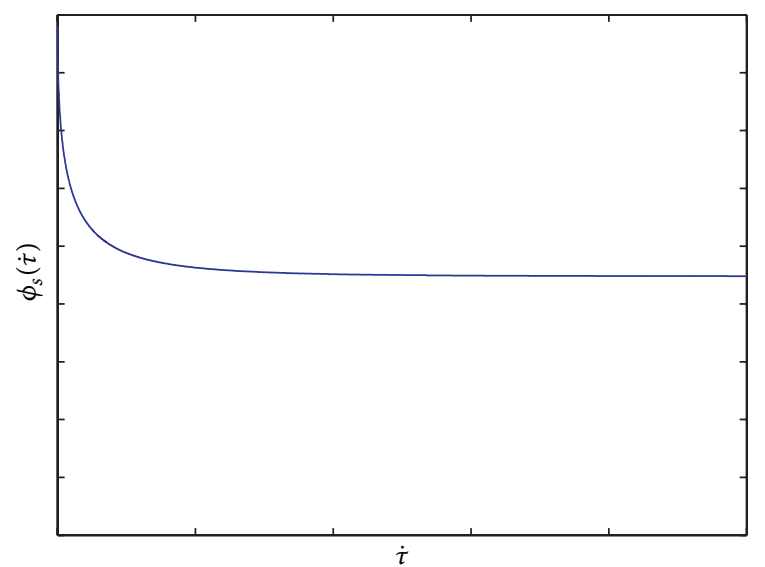

FIGURE 4: Breakaway torque versus rate of change of the applied torque.

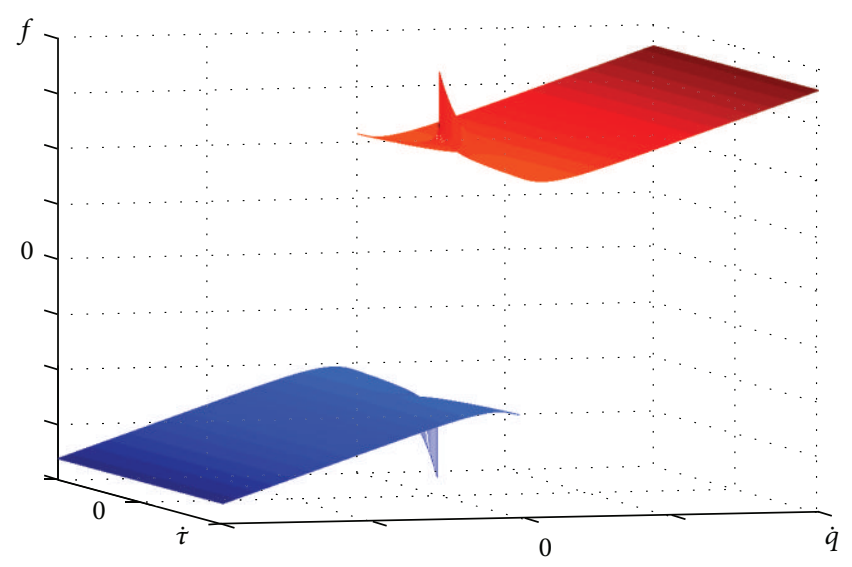

Figure 5: 3D representation of model (19).

Models (19), (20), and (21) are the improved versions of Armstrong model (5) and (6) and LuGre model (11)-(13), respectively; they both include the Stribeck effect, and the dynamic one also shows the effect of microdisplacements. In the following section we explain how to estimate the parameters in these proposed models.

\section{Parameter Identification}

The proposed static model (19) and (20) includes seven parameters $\left(f_{s}, f_{c}, f_{v}, v_{s}, \delta_{v}, t_{s}\right.$, and $\left.\delta_{t}\right)$, and the dynamic model (21) includes two more parameters $\left(\sigma_{0}\right.$ and $\left.\sigma_{1}\right)$. In the literature there exist several methods to obtain $f_{s}, f_{c}$, and $f_{v}$ (see, e.g., $[11,12])$. However, in this paper we propose to obtain the curves of friction against velocity, $f_{k}(\dot{q})$, given by (5), and of breakaway torque against rate of change of the applied torque, $\phi_{s}(\dot{\tau})$, defined in (14), both from experimental data, and then use those curves to estimate the friction parameters. The following subsections explain how to do this.

4.1. Obtention of the Curve $f_{k}(\dot{q})$. In order to get the curve $f_{k}(\dot{q})$ it is necessary to configure the drive of the motor in velocity mode, so that the drive's input voltage is proportional to the desired velocity of the motor. In case that the drive cannot be configured in velocity mode but in torque mode, then a velocity controller with torque output should be designed externally. Moreover, the torque delivered by the motor should be measured in some way (although most of the industrial servomotor/drive systems already include this feature).

The next step is to apply constant velocity references to the drive. Ideally, the actual velocity of the motor should be the same as the desired velocity, but even if this is not the case, it is required to measure both the actual velocity and torque from the motor. Note that these two variables should be constant if so is the desired velocity. And as the acceleration must be zero, then, from (1),

$$
f_{k}(\dot{q})=\tau \text {. }
$$

Thus, the curve $f_{k}(\dot{q})$ should be obtained from the measurements of $\tau$ for different values of $\dot{q}$, from very low to very high velocities.

4.2. Obtention of the Curve $\phi_{S}(\dot{\tau})$. To obtain this curve it is necessary to configure the drive of the motor in torque mode. It is assumed that the drive has an ideal torque controller so that the commanded torque to the drive equals the torque delivered by the motor $\tau$.

We want to find the breakaway torque as a function of the rate of change of the applied torque, that is, $\dot{\tau}$. We only need 
to apply torque ramps with different slope (because if $\tau=m t$, then $\dot{\tau}=m$ ) and measure the value of the torque for which the motor begins to move. After doing this, a graph similar to Figure 4 should be obtained.

4.3. Identification of $f_{s}, f_{c}$, and $f_{v}$. These parameters can be obtained by methods already reported in the literature (see, e.g., $[11,12])$. An alternative is proposed here, for those cases where curves $f_{k}(\dot{q})$ and/or $\phi_{s}(\dot{\tau})$ are obtained experimentally.

It should be noticed that, in the case of $\phi_{s}(\dot{\tau})$, the values of $f_{s}$ and $f_{c}$ can be obtained directly as the bounds of such a curve (see Figure $6(\mathrm{~b})$ ). In the case of the curve $f_{k}(\dot{q})$ we also have a slope due to the effect of the viscous friction (term $\left.f_{v} \dot{q}\right)$, but it is easy to check that, for high velocities, once the Stribeck effect is over, the value of that slope is just $f_{v}$, and the intersection of such ramp with the axis $\dot{q}=0$ gives the value of $f_{c}$ (see Figure 6(a)).

In case that the values of $f_{c}$ and $f_{s}$, obtained from the graphs $f_{k}(\dot{q})$ and $\phi_{s}(\dot{\tau})$ were different, it is recommended to take average values.

4.4. Identification of $v_{s}, t_{s}, \delta_{v}$, and $\delta_{t}$. First, notice that these four parameters determine the way the exponential function decreases from its maximum (at $f_{s}$ ) to its final value in both $f_{k}(\dot{q})$ and $\phi_{s}(\dot{\tau})$. After obtaining the experimental data and determining values of $f_{s}, f_{c}$, and $f_{v}$ (as described in the previous subsection), it is recommended to use a curve fitting software (such as MATLAB's CFTools) to estimate optimum values of $t_{s}$ and $\delta_{t}$ in the case of $\phi_{s}(\dot{\tau})$ and $v_{s}$ and $\delta_{v}$ in the case of $f_{k}(\dot{q})$.

4.5. Identification of $\sigma_{0}$ and $\sigma_{1}$. For the identification of these parameters we use the procedure described in [13], where the behaviour of a motor with friction in the presliding phase is compared to a mass-spring-damper linear system. By linearizing the model of the motor (1) with LuGre friction (11)-(13) we get the following torque to position transfer function:

$$
\frac{q(s)}{\tau(s)}=\frac{1}{J s^{2}+\left(\sigma_{1}+f_{v}\right) s+\sigma_{0}} .
$$

Now consider that a periodic (sinusoidal) torque signal is applied to the drive, configured in torque mode; that is,

$$
\tau=A \sin (\omega t)
$$

with $A$ being a constant lower than the Coulomb friction parameter $f_{c}$ (for better results $A<f_{c} / 2$ is recommended). In such conditions, the rotor performs sinusoidal microdisplacements which can be measured if the motor includes high-resolution encoders. If $\theta_{0}$ is the amplitude of the steadystate microdisplacements, then

$$
\sigma_{0}=\frac{A}{\theta_{0}}
$$

In the case of $\sigma_{1}$ we can see from (23) that this parameter affects the transient response of the linear system. Comparing the denominator of the transfer function (23) with the standard one for a second-order linear system $s^{2}+2 \zeta \omega_{n} s+\omega_{n}^{2}$ we can see that the undamped natural frequency $\omega_{n}$ and the damping coefficient $\zeta$ correspond to $\omega_{n}=\sqrt{\sigma_{0} / J}$ and $\zeta=$ $\left(\sigma_{1}+f_{v}\right) / 2 \sqrt{J \sigma_{0}}$.

Thus, if we can measure the transient response to a step torque of amplitude lower than $f_{c}$, we can estimate the value of $\zeta$, and finally $\sigma_{1}=2 \zeta \sqrt{J \sigma_{0}}-f_{v}$, where it is assumed that the motor's moment of inertia $J$ is known from the data sheet.

\section{Experiments}

In this section we present some experiments carried out on a direct-drive BLDC servomotor, model DM1200A from Parker Compumotor [14]. According to the data sheet this motor has a moment of inertia $J=0.167 \mathrm{Kgm}^{2}$, and it has a high-resolution encoder of 1024000 pulses per revolution (so it is possible to measure a deviation of 0.00035 degrees). First, we explain how we applied the procedures described in the previous section to compute the friction parameters of the motor. After that, we include some experiments which allow the validation of the proposed model.

5.1. Curve Fitting and Parameter Estimation. Experiments were carried out for the determination of $f_{k}(\dot{q})$ and $\phi_{s}(\dot{\tau})$ following the procedures mentioned in Sections 4.1 and 4.2. The parameters that better fit the experimental data to the curves (5) and (14) were determined. To do this, the software CFTools from MATLAB was used. Figures 7 and 8 show the experimental data and the fitted curves for both cases.

The parameters obtained with CFTools are shown in Table 1. The columns "Value (+)" and "Value (-)" in that table show the values of the parameters obtained in the positive (counter clock wise) and negative (clock wise) directions of the rotor motion, respectively. From this, it is observed that friction is not symmetric.

5.2. Model Validation. To verify the benefit of using friction models with the modification proposed in Section 3 against classical friction models (with $f_{s}$ constant), we performed simulations using different friction models and torque inputs. The friction models under analysis were the following.

(i) Coulomb + viscous friction $(\mathrm{C}+\mathrm{V}),(4)$.

(ii) Improved static + Coulomb + viscous friction $(\mathrm{I}(\mathrm{S}+$ $\mathrm{C}+\mathrm{V})),(4)$ and (6).

(iii) Static + Armstrong friction $(S+A),(5)$ and (6).

(iv) Improved static + Armstrong friction $(\mathrm{I}(\mathrm{S}+\mathrm{A}))$, (19) and (20).

(v) LuGre friction (LG), (11)-(13).

(vi) Improved LuGre friction (I(LG)), (21).

Figures 9, 10, and 11 show the velocity response obtained from simulations and experiments with the real motor to different torque inputs. Figure 9 shows the velocity for a ramp torque input of $\tau=t \mathrm{Nm}$; Figure 10 shows the result for a different ramp torque input; $\tau=0.05 t$. 


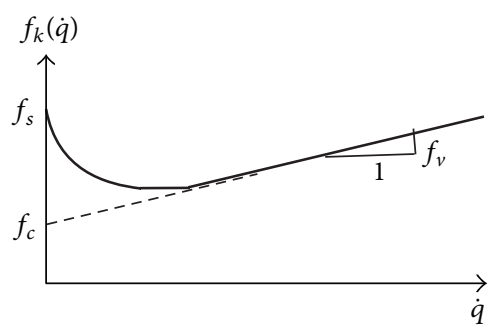

(a)

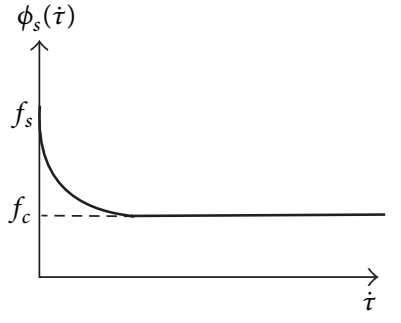

(b)

Figure 6: Reference curves used to get the parameters $f_{s}, f_{c}$, and $f_{v}$, from (a) $f_{k}(\dot{q})$ and (b) $\phi_{s}(\dot{\tau})$.

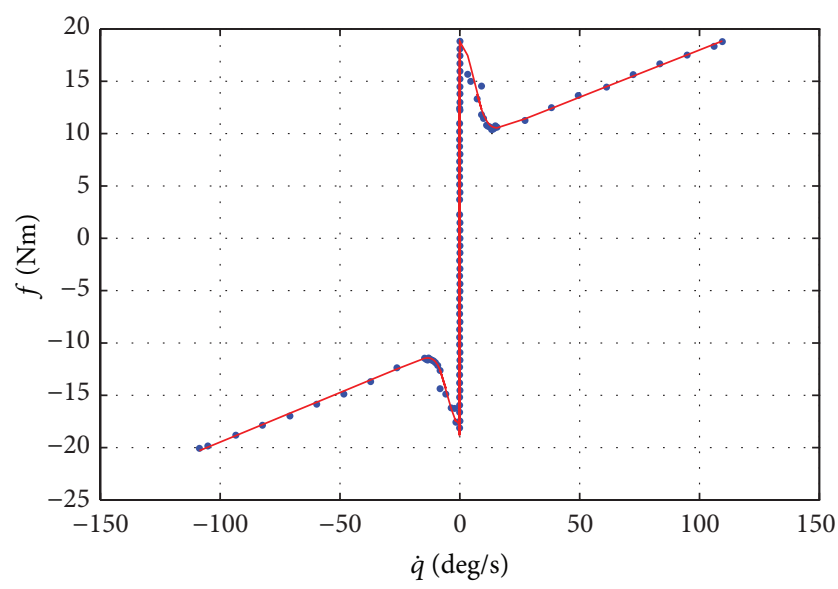

FIGURE 7: Experimental data and fitted curve for $f_{k}(\dot{q})$.

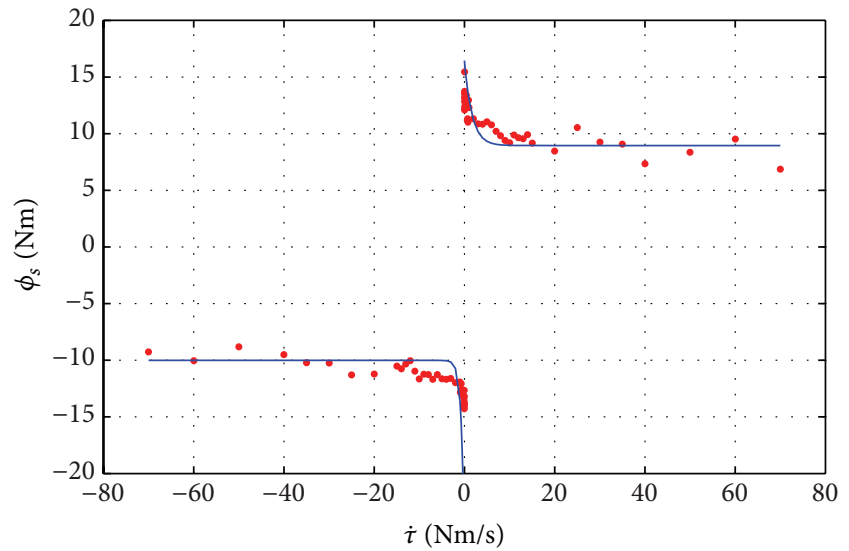

FIGURE 8: Experimental data and fitted curve for $\phi_{s}(\dot{\tau})$.

It can be clearly observed in these figures that those models including the modification proposed in this paper have a response in simulation which agrees better with the experimental results, especially with respect to time in which the breakaway point is reached.

In Figure 10, for example, we can see that the breakaway force for the $\mathrm{C}+\mathrm{V}$ model is reached at about 180 seconds; for the $S+A$ and LG models (these using $f_{s}$ as a constant) we get about 360 seconds, and those models with the improvement

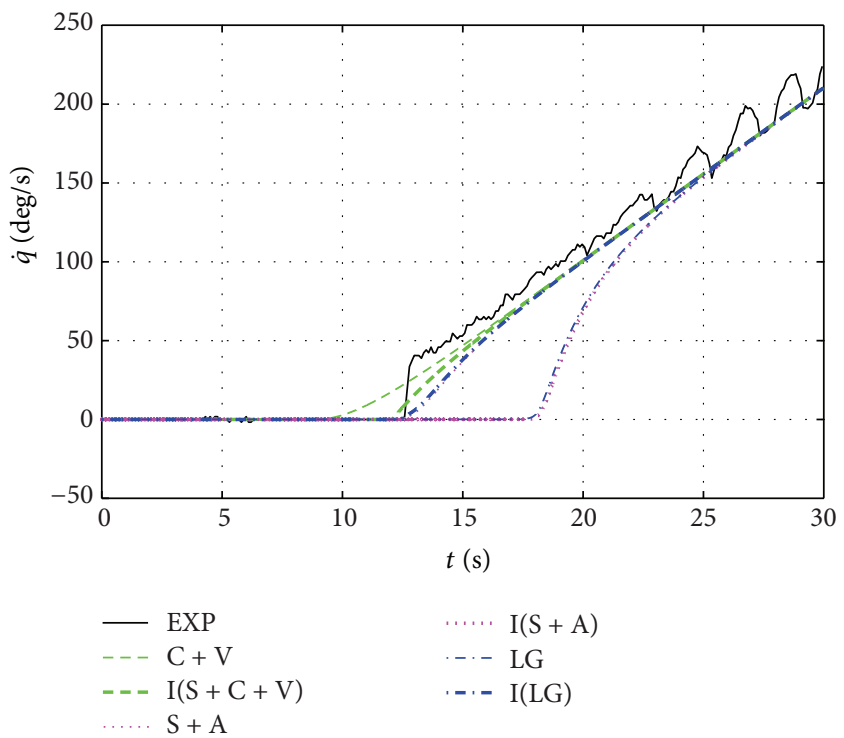

FIgURE 9: Response to a torque input $\tau=m t$, with $m=1 \mathrm{Nm} / \mathrm{s}$.

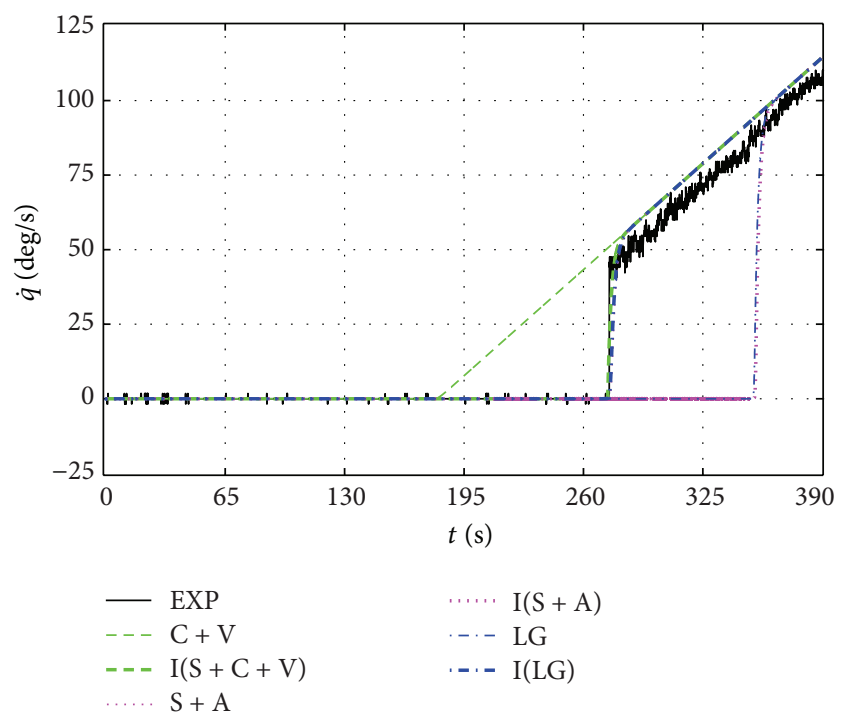

FIGURE 10: Response to a torque input $\tau=m t$, with $m=0.05 \mathrm{Nm} / \mathrm{s}$. 


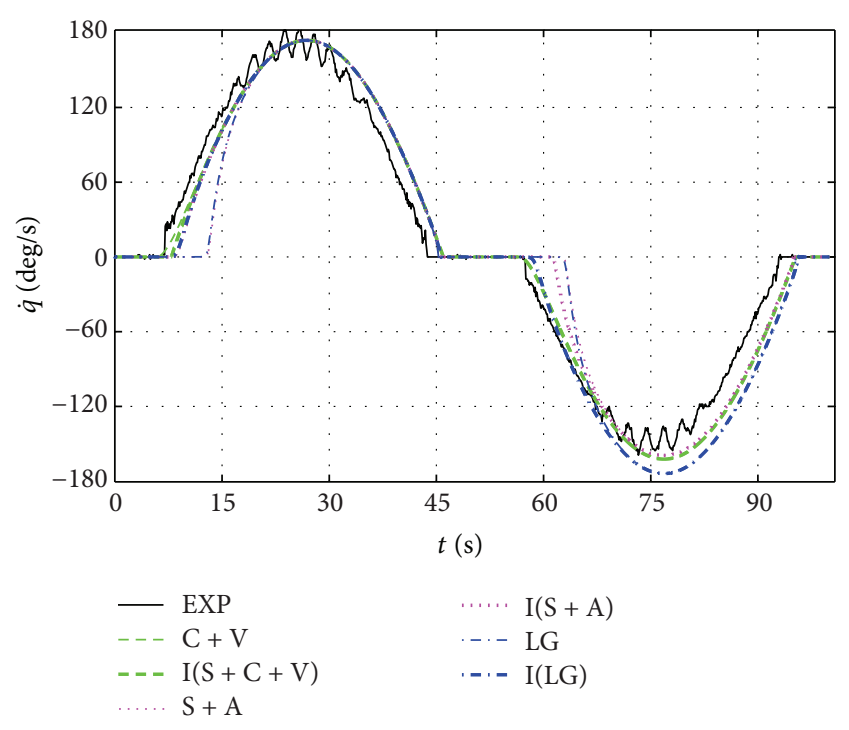

FIGURE 11: Response to a torque input $\tau=25 \sin (2 \pi 0.01 t) \mathrm{Nm}$.

TABLE 1: Estimated friction parameters.

\begin{tabular}{|c|c|c|c|}
\hline Parameter & Value $(+)$ & Value $(-)$ & Unit \\
\hline$f_{c}$ & 8.955 & 10.01 & $\mathrm{Nm}$ \\
\hline$f_{v}$ & 5.234 & 5.335 & $\mathrm{Nms} / \mathrm{rad}$ \\
\hline$f_{s}$ & 17.617 & 23.813 & $\mathrm{Nm}$ \\
\hline$v_{s}$ & 0.145 & 0.078 & $\mathrm{rad} / \mathrm{s}$ \\
\hline$t_{s}$ & 0.888 & 1.954 & $\mathrm{Nm} / \mathrm{s}$ \\
\hline$\delta_{v}$ & 1.032 & 1.286 & \\
\hline$\delta_{t}$ & 0.172 & 0.287 & \\
\hline$\sigma_{0}$ & \multicolumn{2}{|c|}{17260} & $\mathrm{Nm} / \mathrm{rad}$ \\
\hline$\sigma_{1}$ & \multicolumn{2}{|c|}{66.708} & $\mathrm{Nms} / \mathrm{rad}$ \\
\hline
\end{tabular}

$(\mathrm{I}(\mathrm{S}+\mathrm{C}+\mathrm{V}), \mathrm{I}(\mathrm{S}+\mathrm{A})$, and $\mathrm{I}(\mathrm{LG}))$ are closer to the experimental value of 273.7 seconds.

Finally, Figure 11 shows the resultant velocity for a sinusoidal input $\tau=25 \sin (2 \pi(0.01) t)$ with an amplitude exceeding the maximum static friction coefficient. A similar analysis to that of Figures 9 and 10 can be done for Figure 11.

\section{Conclusions}

Friction occurs whenever there is a relative motion between two bodies. The classic static friction models consider the maximum static friction coefficient $f_{s}$ as a constant. In this paper we propose to use a function $\phi_{s}(\dot{\tau})$ instead of the $f_{s}$ coefficient. We first define an expression that characterizes such function, and then we replace it in the classic friction models containing the $f_{s}$ coefficient. New friction models are thus obtained, and, in order to validate them, some experiments on a motor were carried out. As a result we have that those models with the proposed modification have a behavior which is closer to the reality.

\section{Acknowledgments}

This work was partially supported by DGEST and CONACYT Mexico (Grant 60230).

\section{References}

[1] H. Olsson, Control of systems with friction [Ph.D. thesis], Lund Institute of Technology, Lund, Sweden, 1996.

[2] J. Courtney-Pratt and E. Eisner, "The effect of a tangential force on the contact of metallic bodies," Proceedings of the Royal Society A, vol. 238, pp. 529-550, 1957.

[3] E. Rabinowicz, "Stick and slip," Scientific American, vol. 194, pp. 109-118, 1956.

[4] V. I. Johannes, M. A. Green, and C. A. Brockley, "The role of the rate of application of the tangential force in determining the static friction coefficient," Wear, vol. 24, no. 3, pp. 381-385, 1973.

[5] R. Campa, E. Torres, F. Salas, and V. Santibáñez, “On modeling and parameter estimation of brushless DC servoactuators for position control tasks," in Proceedings of the 17th International Federation of Automatic Control World Congress, Seoul, Korea, 2008.

[6] A. Morin, "New friction experiments carried out at Metz in 18311833," in Proceedings of the French Royal Academy of Sciences, vol. 4, pp. 1-128, 1833.

[7] R. Stribeck, "The key qualities of sliding and roller bearings," Zeitschrift Des Vereins Deutscher Ingenieure, vol. 46, pp. 13421348, 1902.

[8] B. Armstrong-Helouvry, Control of Machines With Friction, Kluwer Academic Publishers, 1991.

[9] P. Dahl, “A solid friction model," Tech. Rep. TOR-0158(3107-18)1, The Aerospace Corporation, El Segundo, Calif, USA, 1968.

[10] C. C. de Wit, H. Olsson, K. J. Åström, and P. Lischinsky, "A new model for control of systems with friction," IEEE Transactions on Automatic Control, vol. 40, no. 3, pp. 419-425, 1995.

[11] J. Swevers, F. Al-Bender, C. G. Ganseman, and T. Prajogo, "An integrated friction model structure with improved presliding behavior for accurate friction compensation," IEEE Transactions on Automatic Control, vol. 45, no. 4, pp. 675-686, 2000.

[12] R. Kelly, J. Llamas, and R. Campa, "A measurement procedure for viscous and Coulomb friction," IEEE Transactions on Instrumentation and Measurement, vol. 49, no. 4, pp. 857-861, 2000.

[13] K. J. Åström and C. Canudas-de-Wit, "Revisiting the LuGre friction model," IEEE Control Systems Magazine, vol. 28, no. 6, pp. 101-114, 2008.

[14] Parker Motion \& Control Dynaserv DM \& DR Direct Drive Servos User Guide, 1995, Compumotor Division, p/n 88-013940$01 \mathrm{~B}$. 


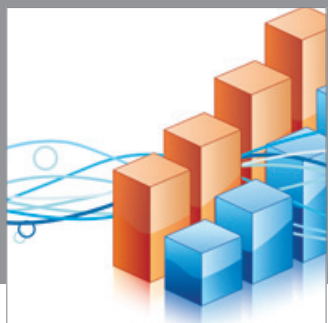

Advances in

Operations Research

mansans

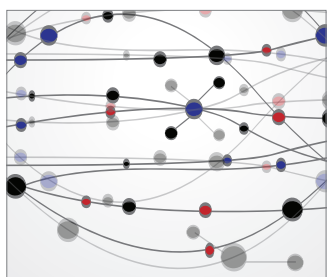

The Scientific World Journal
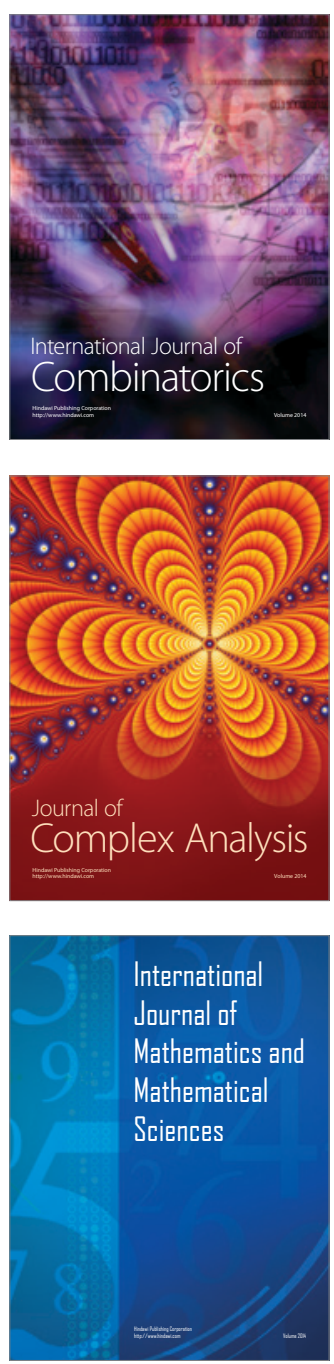
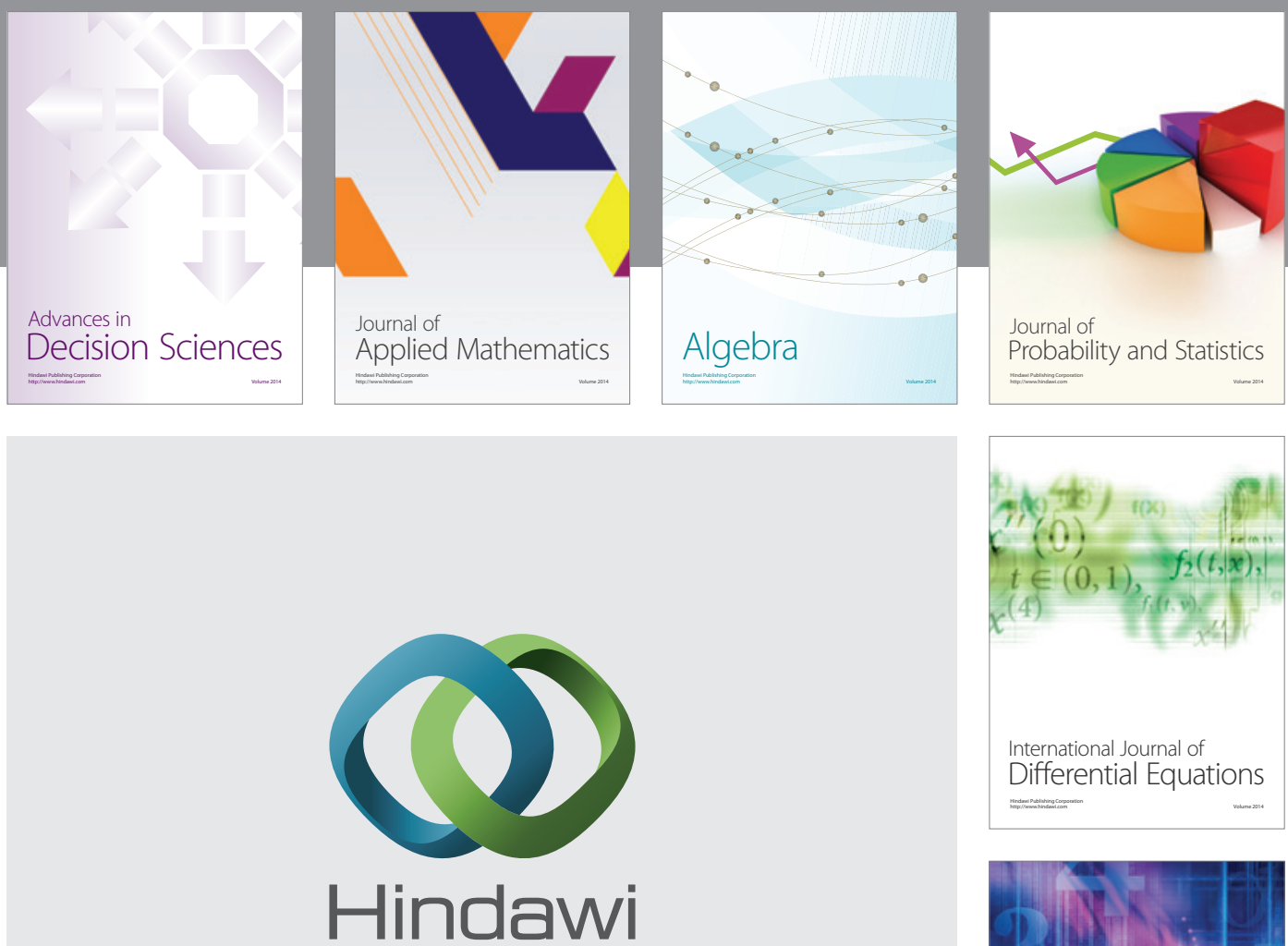

Submit your manuscripts at http://www.hindawi.com
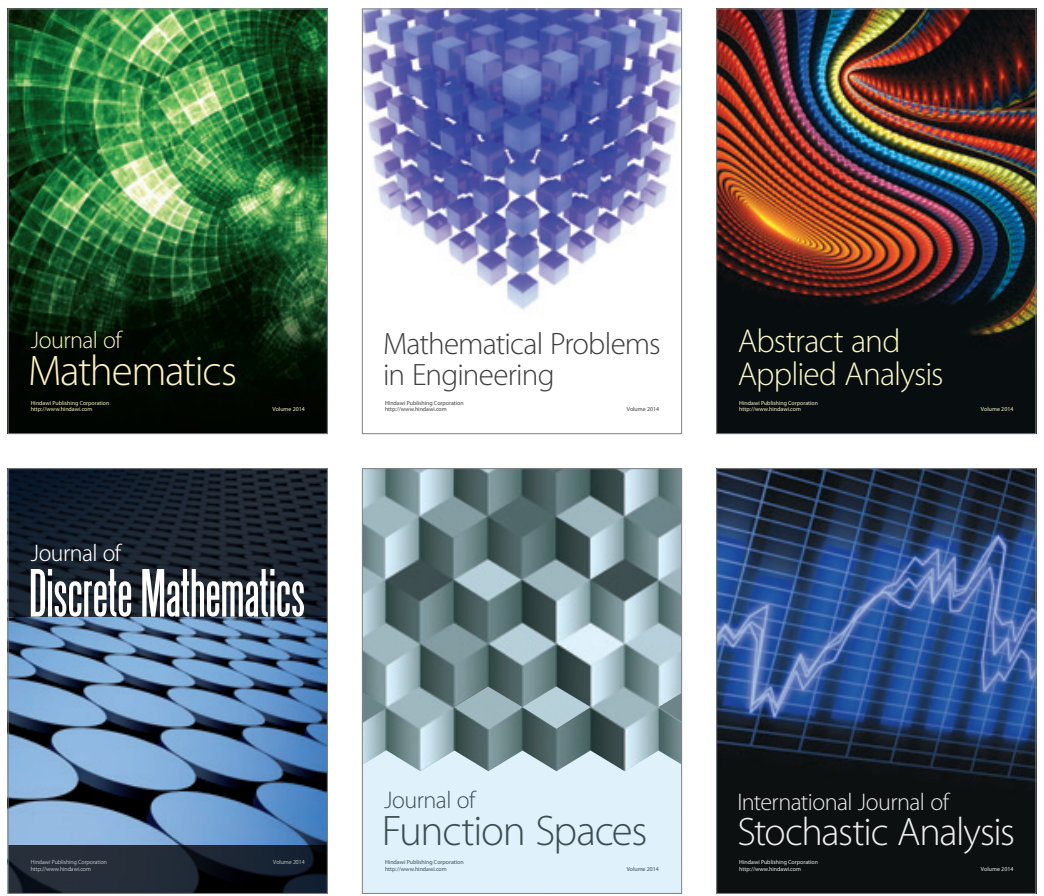

Journal of

Function Spaces

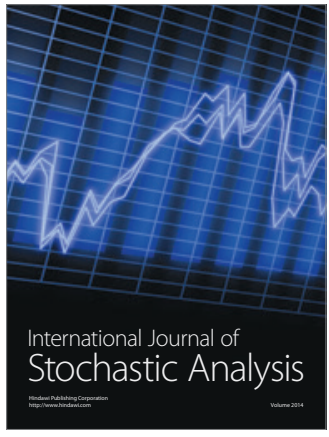

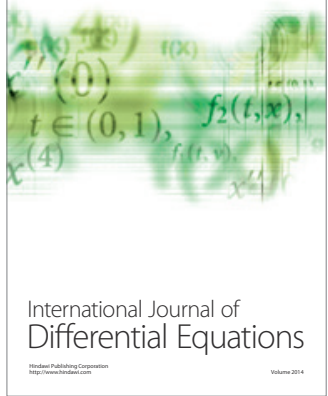
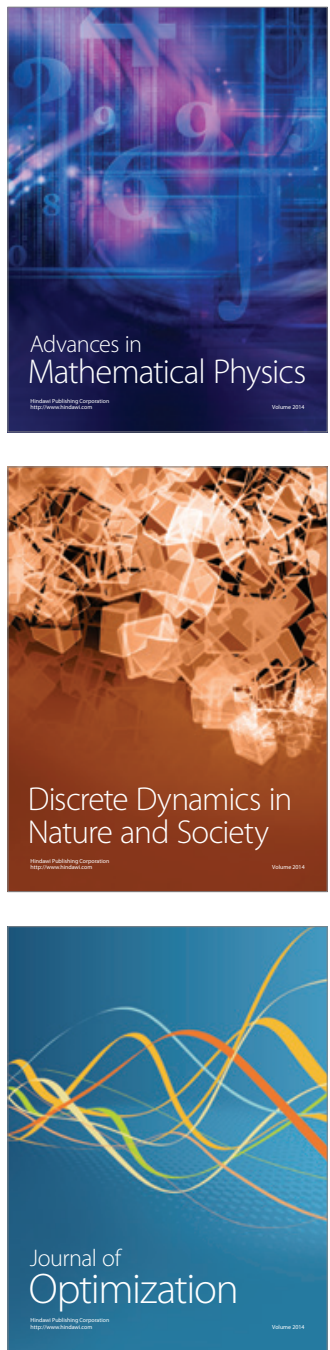